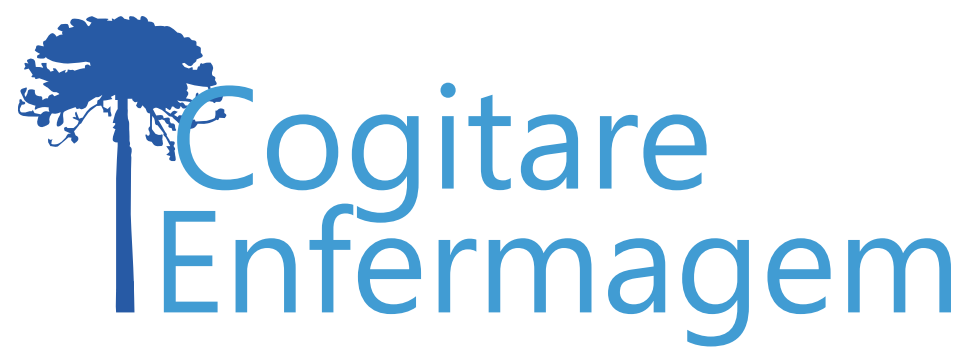

\title{
MULTIDIMENSIONALIDADE DA GRAVIDADE DOS TRANSTORNOS RELACIONADOS A SUBSTÂNCIAS PSICOATIVAS PELO ADDICTION SEVERITY INDEX-6
}

\begin{abstract}
Manuela Kaled', Fernanda Carolina Capistrano², Aline Cristina Zerwes Ferreira ${ }^{3}$, Mariluci Alves Maftum ${ }^{4}$, Maria de Fátima Mantovani ${ }^{5}$, Rosibeth Del Carmen Munoz Palm ${ }^{6}$, Camila Bonfim de Alcântara ${ }^{7}$
\end{abstract}

\section{RESUMO}

Objetivo: caracterizar a gravidade dos transtornos relacionados a substâncias nas pessoas em tratamento nos Centros de Atenção Psicossocial Alcool e outras Drogas.

Método: estudo transversal, realizado em três Centros de Atenção Psicossocial Álcool e outras Drogas III, em Curitiba-Paraná, com 137 pessoas com transtornos relacionados a substâncias. Os dados foram coletados entre abril e novembro de 2018, mediante a aplicação do instrumento Addiction Severity Index sexta versão, e analisados descritivamente a partir de frequências absolutas e relativas, média e desvio padrão.

Resultados: do total da amostra, 89,1\% (122), eram do sexo masculino; as subescalas de maior gravidade foram Família/crianças 57,8 ( \pm 8,6), Álcool 52,8 ( $(10,9)$ e Família/problemas sociais 51,0 $( \pm 8,9)$.

Conclusão: $\bigcirc$ uso de substâncias psicoativas afeta as relações familiares e sociais e o álcool, uma substância lícita, de fácil acesso, está presente na vida das pessoas desde a infância, acabando por ter maior frequência entre todas as faixas etárias.

DESCRITORES: Gravidade do Paciente; Transtornos Relacionados ao Uso de Substâncias; Drogas llícitas; Serviços de Saúde Mental; Saúde Mental.

\footnotetext{
*Artigo extraído da dissertação de mestrado "Gravidade dos transtornos relacionados a substâncias nas pessoas em tratamento em Centros de Atenção Psicossocial de álcool e outras drogas". Universidade Federal do Paraná, 2019.

COMO REFERENCIAR ESTE ARTIGO:

Kaled M, Capistrano FC, Ferreira ACZ, Maftum MA, Mantovani M de F, Palm R Del CM, et al. Multidimensionalidade da gravidade dos transtornos relacionados a substâncias psicoativas pelo Addiction Severity Index-6. Cogitare enferm. [Internet]. 2020 [acesso em "colocar data de acesso, dia, mês abreviado e ano"]; 25. Disponível em: http://dx.doi.org/10.5380/ce.v25i0.70636.
}

${ }^{1}$ Enfermeira. Mestranda em Enfermagem. Universidade Federal do Paraná. Curitiba, PR, Brasil.

${ }^{2}$ Enfermeira. Doutora em Enfermagem. Enfermeira do Núcleo de Saúde Mental da Secretaria de Saúde de São José dos Pinhais. São José dos Pinhais, PR, Brasil.

${ }^{3}$ Enfermeira. Doutoranda em Enfermagem. Universidade Federal do Paraná. Curitiba, PR, Brasil. ()

${ }^{4}$ Enfermeira. Doutora em Enfermagem. Docente de Pós-Graduação em Enfermagem da Universidade Federal do Paraná. Curitiba, PR, Brasil. 9

${ }^{5}$ Enfermeira. Doutora em Enfermagem. Docente de Pós-Graduação em Enfermagem da Universidade Federal do Paraná. Curitiba, PR, Brasil.

${ }^{6}$ Terapeuta Ocupacional. Doutora em Enfermagem. Docente da Universidade Federal do Paraná. Curitiba, PR, Brasil. (3)

${ }^{7}$ Discente de Enfermagem. Universidade Federal do Paraná. Curitiba, PR, Brasil. 


\title{
MULTIDIMENSIONALITY OF SEVERITY OF PSYCHOACTIVE SUBSTANCES RELATED TO ADDICTION SEVERITY INDEX-6
}

\begin{abstract}
Objective: to characterize the severity of substance-related disorders in patients undergoing treatment at the Psychosocial Care Centers for Alcohol and other Drugs.

Method: a transversal study conducted in three Psychosocial Care Centers for Alcohol and other Drugs III, in Curitiba-Paraná, with 137 people with substance-related disorders. Data were collected from April to November of 2018, using the Addiction Severity Index sixth version, and analyzed descriptively using absolute and relative frequencies, mean and standard deviation.

Results: out of the entire sample, $89.1 \%$ (122) were male; the most severe subscales were Family/children 57.8 ( \pm 8.6), Alcohol 52.8 ( \pm 10.9 ) and Family/social problems $51.0( \pm 8.9$ ).

Conclusion: The use of psychoactive substances affects family and social relatedness, and alcohol, a legal substance, easily accessible, has been present in people's lives since childhood, ending up being more frequent among all age groups.
\end{abstract}

DESCRIPTORS: Patient Acuity; Substance-Related Disorders; Illicit drugs; Mental Health Services; Mental Health.

\section{CARÁCTER MULTIDIMENSIONAL DE LA GRAVEDAD DE LOS TRASTORNOS ASOCIADOS A SUSTANCIAS PSICOACTIVAS POR EL ADDICTION SEVERITY INDEX-6}

\begin{abstract}
RESUMEN:
Objetivo: caracterizar la gravedad de los trastornos asociados a sustancias en las personas en tratamiento en los Centros de Atención Psicosocial Alcohol y otras Drogas.

Método: estudio transversal, que se realizó en tres Centros de Atención Psicosocial Alcohol y otras Drogas III, en Curitiba-Paraná, con 137 personas con trastornos asociados a sustancias. Se obtuvieron los dados entre abril y noviembre de 2018, por medio de aplicación del instrumento Addiction Severity Index sexta versión, analizándolos de modo descriptivo a partir de frecuencias absolutas y relativas, promedio y desviación estándar.

Resultados: del total de la muestra, 89,1\% (122) eran del sexo masculino; las sub escalas de mayor gravedad fueron Familia/niños $57,8( \pm 8,6)$, Alcohol 52,8 $( \pm 10,9)$ y Familia/problemas sociales $51,0( \pm 8,9)$.

Conclusión: El uso de sustancias psicoactivas afecta las relaciones familiares y sociales y el alcohol, una substancia lícita, de fácil acceso, está presente en la vida de las personas desde la infancia, presentando mayor frecuencia entre todas las franjas etarias.
\end{abstract}

DESCRIPTORES: Gravedad del Paciente; Trastornos Asociados al Uso de Sustancias; Drogas Ilícitas; Servicios de Salud Mental; Salud Mental. 
O consumo de substâncias psicoativas (SPA) é um problema mundial de saúde pública e suas consequências são descritas desde a antiguidade ${ }^{(1-3)}$. Sabe-se que 271 milhões de pessoas, 5,5\% da população mundial, utilizaram SPA alguma vez no ano de 2016, dessas, 35 milhões foram consideradas consumidoras problemas e apresentaram consequências nocivas devido ao uso ${ }^{(1)}$.

No Brasil, o último Levantamento Nacional de Álcool e Drogas (LENAD) apresentou que sete milhões $(6,8 \%)$ da população consumiu cannabis alguma vez na vida, cinco milhões $(3,8 \%)$ derivados de cocaína, três milhões $(2,7 \%)$ outros estimulantes, e dois milhões $(2,2 \%)$ solventes ${ }^{(4)}$. Contudo, um dos agravantes do consumo excessivo é o desenvolvimento de transtornos relacionados a substâncias que, por sua vez, podem desencadear consequências de diferentes gravidades no âmbito social, econômico e político da pessoa, família e sociedade ${ }^{(4,5)}$.

Assim, pesquisadores desenvolveram o Addicction Severity Index (ASI-6), um instrumento para quantificar e qualificar a gravidade das consequências decorrentes dos transtornos relacionados a substâncias ${ }^{(6)}$, que foi validado no Brasil, em estudo com 740 indivíduos, obtendo alfa de Cronbach entre 0,64 e 0,93. O ASI-6 é utilizado em pesquisas da temática dos transtornos relacionados a substâncias, pois possibilita avaliar o paciente de modo multidimensional, identificando a gravidade devida ao uso abusivo de SPA nas áreas: clínica/médica; emprego e sustento; álcool; drogas; legal; sociofamiliar e saúde mental/psiquiátrica ${ }^{(5)}$.

Considerando tais áreas afetadas pelo uso abusivo de substâncias, assim como o intenso aumento do consumo e os impactos negativos que acarretam à vida da pessoa, família e sociedade, e que raramente se manifestam em episódio individual, afetando para além dos relacionamentos. Assim, desenvolver estudos sobre a gravidade dos transtornos relacionados a substâncias poderá contribuir com o aprimoramento de políticas públicas, gestão, profissionais da área da saúde, em especial da saúde mental, nas práticas de cuidados aos usuários e família, possibilitando minimizar a gravidade situacional.

Este estudo teve como objetivo caracterizar a gravidade dos transtornos relacionados a substâncias nas pessoas em tratamento nos Centros de Atenção Psicossocial Álcool e outras Drogas.

\section{MÉTODO}

Pesquisa transversal, realizada em três Centros de Atenção Psicossocial Álcool e outras Drogas III (CAPS AD III) do município de Curitiba-PR, Brasil, com pessoas com transtornos relacionados a substâncias. A amostra foi por conveniência e foram incluídos aqueles que estavam em tratamento e com idade mínima de 18 anos. Os critérios de exclusão foram: comprometimento cognitivo, identificado pela equipe de saúde e/ou registrado em prontuário, e estar em estado de intoxicação sem condições de responder.

No período da coleta de dados, 1013 pessoas estavam cadastradas, 174 foram abordadas, 10 excluídas (nove por comprometimento cognitivo e uma por intoxicação por álcool), 24 se recusaram a participar, três desistiram após início da entrevista, resultando em uma amostra de 137 pessoas.

A coleta de dados ocorreu entre abril e novembro de 2018, pela aplicação do instrumento Addiction Severity Index (ASI 6), por meio de uma entrevista semiestruturada com 252 questões distribuídas em sete diferentes áreas. 
Os dados coletados foram inseridos em uma base de dados online e, após, exportados para o programa computacional Statistical Package for the Social Sciences versão 21 para análise estatística descritiva. Na análise, as variáveis contínuas foram descritas em médias e desvio padrão e as variáveis categóricas através de frequências absolutas e relativas.

Para o cálculo de escores sumários do ASI-6, foi utilizado o método descrito por Kessler, que explicita que para gerar os escores é necessário utilizar os Escores Sumários de Funcionamento Recente do ASI-6 (SS-R), que os padroniza segundo o T-score, que estabelece uma média de 50 e desvio padrão de 10, com variação do escore entre 0 e 100 , sendo que maiores pontuações indicam maior gravidade ${ }^{(5)}$.

Este artigo é proveniente dos dados de uma dissertação, que inclui o projeto maior aprovado pelo Comitê de Ética em Pesquisa da Universidade Federal do Paraná com o parecer $n^{\circ}$ 2.033.006 e Comitê de Ética da Secretaria Municipal de Saúde de Curitiba parecer $n^{\circ} 2.071 .35$.

\section{RESULTADOS}

Da amostra de 137 pessoas (Tabela 1), 89,1\% (122) eram do sexo masculino, a média da idade foi 41,7 anos $( \pm 12,5), 46,0 \%$ (63) declararam-se brancos e 41,6\% (57) estavam solteiros. A escolaridade foi de 44,5\% (61) com até o Ensino Fundamental. Quanto às questões socioeconômicas, $38 \%$ (52) relataram estar desempregado e procurando emprego e a renda mensal menor que um salário mínimo foi relatada por $87,6 \%(120)$ das pessoas.

Tabela 1 - Características socioeconômicas das pessoas em tratamento. Curitiba, PR, 2018 (continua)

\begin{tabular}{lcccccc} 
Variáveis & \multicolumn{2}{c}{ Masculino } & \multicolumn{2}{c}{ Feminino } & \multicolumn{2}{c}{ Total } \\
\cline { 2 - 7 } & $\mathbf{N}$ & $(\%)$ & $\mathbf{N}$ & $(\%)$ & $\mathbf{N}$ & $(\%)$ \\
\hline Sexo & 122 & $(89,1)$ & 15 & $(10,9)$ & 137 & $(100)$ \\
\hline Idade & $41,7 \pm 12,3$ & $41,1 \pm 14,1$ & $41,7 \pm 12,5$ \\
\hline$\leq 29$ anos & 24 & $(17,5)$ & 4 & $(2,9)$ & 28 & $(20,4)$ \\
\hline $30-49$ anos & 62 & $(45,3)$ & 6 & $(4,4)$ & 68 & $(49,6)$ \\
\hline$\geq 50$ anos & 36 & $(26,3)$ & 5 & $(3,6)$ & 41 & $(30)$ \\
\hline Raça & & & & & & \\
\hline Negra & 12 & $(8,8)$ & 1 & $(0,7)$ & 13 & $(9,5)$ \\
\hline Branca & 52 & $(38)$ & 11 & $(8)$ & 63 & $(46)$ \\
\hline Amarela/oriental & 6 & $(4,4)$ & 1 & $(0,7)$ & 7 & $(5,1)$ \\
\hline Parda & 50 & $(36,5)$ & 2 & $(1,5)$ & 52 & $(38)$ \\
\hline Indígena & 2 & $(1,4)$ & 0 & $(0)$ & 2 & $(1,4)$ \\
\hline Estado Conjugal & & & & & & \\
\hline Solteiro & 50 & $(36,5)$ & 7 & $(5,1)$ & 57 & $(41,6)$ \\
\hline Casado & 12 & $(8,8)$ & 1 & $(0,7)$ & 13 & $(9,5)$ \\
\hline União Consensual & 8 & $(5,8)$ & 3 & $(2,2)$ & 11 & $(8)$ \\
\hline Viúvo & 3 & $(2,2)$ & 2 & $(1,5)$ & 5 & $(3,6)$ \\
\hline
\end{tabular}




\begin{tabular}{lcccccc}
\hline Divorciado & 16 & $(11,8)$ & 0 & $(0)$ & 16 & $(11,8)$ \\
\hline Separado & 33 & $(24,1)$ & 2 & $(1,5)$ & 35 & $(25,5)$ \\
\hline Escolaridade & & & & & & \\
\hline Ensino Fundamental & 55 & $(40,1)$ & 6 & $(4,4)$ & 61 & $(44,5)$ \\
\hline Ensino Médio & 39 & $(28,5)$ & 8 & $(5,8)$ & 47 & $(34,3)$ \\
\hline Ensino Superior & 5 & $(3,6)$ & 0 & $(0)$ & 5 & $(3,6)$ \\
\hline Nenhum & 23 & $(16,9)$ & 1 & $(0,7)$ & 24 & $(17,6)$ \\
\hline Situação Empregatícia & & & & & & \\
\hline Turno integral & 13 & $(9,5)$ & 0 & $(0)$ & 13 & $(9,5)$ \\
\hline Meio turno & 3 & $(2,2)$ & 0 & $(0)$ & 3 & $(2,2)$ \\
\hline Desempregado / procurando emprego & 46 & $(33,6)$ & 6 & $(4,4)$ & 52 & $(38)$ \\
\hline Fora do mercado / não procura emprego & 48 & $(35)$ & 7 & $(5,1)$ & 55 & $(40,1)$ \\
\hline Trabalho formal/ trabalho informal & 12 & $(8,8)$ & 2 & $(1,4)$ & 14 & $(10,2)$ \\
\hline Renda* & & & & & 14 & $(87,6)$ \\
\hline < Salário Mínimo & 106 & $(77,4)$ & 14 & $(10,2)$ & 120 & $(12,4)$ \\
\hline > Salário Mínimo & 16 & $(11,7)$ & 1 & $(0,7)$ & 17 & \\
*Salário mínimo vigente R\$998,00. & & & & & & $(16)$
\end{tabular}

Sobre as condições físicas dos participantes (Tabela 2), quanto ao sistema circulatório, $22 \%$ (30) relataram ter Hipertensão Arterial Sistêmica. Referente às condições mentais, $32,1 \%$ (44) apresentaram comorbidades, sendo que nos últimos 30 dias 55,5\% (76) se sentiram deprimidos e 61,4\% (84) apresentaram sintomas de ansiedade. Quanto às alterações de comportamento, nos últimos 30 dias, 9,5\% (13) apresentaram agressividade, $18,9 \%$ (26) histórico de ideação suicida, 8,1\% (11) tentativa de suicídio. Quanto a eventos traumáticos, alguma vez durante a vida, 10,9\% (15) sofreram abusos sexuais, 37,3\% (51) foram vítimas de crimes violentos como agressões e espancamentos e $55,5 \%$ (76) estiveram em situações de risco de vida.

Tabela 2 - Caracterização clínica das condições físicas e mentais das pessoas em tratamento. Curitiba, PR, 2018 (continua)

\begin{tabular}{|c|c|c|c|c|c|c|}
\hline \multirow[t]{3}{*}{ Variáveis } & \multicolumn{2}{|c|}{ Masculino } & \multicolumn{2}{|c|}{ Feminino } & \multicolumn{2}{|c|}{ Total } \\
\hline & $\mathbf{N}$ & (\%) & $\mathbf{N}$ & $(\%)$ & $\mathbf{N}$ & (\%) \\
\hline & 122 & $(89,1)$ & 15 & $(10,9)$ & 137 & $(100)$ \\
\hline \multicolumn{7}{|c|}{ Condições Clínicas Físicas } \\
\hline Sistema circulatório & 26 & $(19)$ & 4 & $(2,9)$ & 30 & $(22)$ \\
\hline Sistema neurológico & 16 & $(11,7)$ & 1 & $(0,7)$ & 17 & $(12,4)$ \\
\hline Sistema imunológico & 11 & (8) & 1 & $(0,7)$ & 12 & $(8,7)$ \\
\hline Sistema respiratório & 11 & (8) & 1 & $(0,7)$ & 12 & $(8,7)$ \\
\hline Não se aplica & 58 & $(42,3)$ & 8 & $(5,8)$ & 66 & $(48,2)$ \\
\hline
\end{tabular}


Condições Clínicas Mentais

\begin{tabular}{|c|c|c|c|c|c|c|}
\hline Sim & 37 & (27) & 7 & $(5,1)$ & 44 & $(32,1)$ \\
\hline Não & 85 & (62) & 8 & $(5,8)$ & 93 & $(67,9)$ \\
\hline \multicolumn{7}{|c|}{ Humor deprimido } \\
\hline Sim & 66 & $(48,2)$ & 10 & $(7,3)$ & 76 & $(55,5)$ \\
\hline Não & 20 & $(14,6)$ & 3 & $(2,2)$ & 23 & $(16,8)$ \\
\hline Sob efeito SPA & 11 & $(8)$ & 1 & $(0,7)$ & 12 & $(8,8)$ \\
\hline Não se aplica & 25 & $(18,2)$ & 1 & $(0,7)$ & 26 & $(19)$ \\
\hline \multicolumn{7}{|l|}{ Ansiedade } \\
\hline Sim & 75 & $(54,9)$ & 9 & $(6,5)$ & 84 & $(61,4)$ \\
\hline Não & 15 & $(10,9)$ & 2 & $(1,4)$ & 17 & $(12,3)$ \\
\hline Sob efeito SPA & 8 & $(5,8)$ & 2 & $(1,4)$ & 10 & $(7,3)$ \\
\hline Não se aplica & 24 & $(17,5)$ & 2 & $(1,4)$ & 26 & $(19)$ \\
\hline \multicolumn{7}{|l|}{ Agressividade } \\
\hline Sim & 13 & $(9,5)$ & - & - & 13 & $(9,5)$ \\
\hline Não & 53 & $(38,7)$ & 6 & $(4,4)$ & 59 & $(43,1)$ \\
\hline Sob efeito SPA & 3 & $(2,2)$ & - & & 3 & $(2,2)$ \\
\hline Não se aplica & 53 & $(38,7)$ & 9 & $(6,5)$ & 62 & $(45,2)$ \\
\hline \multicolumn{7}{|l|}{ Ideação suicida } \\
\hline Sim & 24 & $(17,5)$ & 2 & $(1,4)$ & 15 & $(18,9)$ \\
\hline Não & 53 & $(38,7)$ & 9 & $(6,6)$ & 62 & $(45,3)$ \\
\hline Sob efeito SPA & 3 & $(2,2)$ & 0 & 0 & 3 & $(2,2)$ \\
\hline Não se aplica & 42 & $(30,7)$ & 4 & $(2,9)$ & 46 & $(33,6)$ \\
\hline \multicolumn{7}{|l|}{ Tentativa suicídio } \\
\hline Sim & 9 & $(6,6)$ & 2 & $(1,5)$ & 11 & $(8,1)$ \\
\hline Não & 48 & (35) & 8 & $(5,8)$ & 56 & $(40,8)$ \\
\hline Sob efeito SPA & 2 & $(1,5)$ & 1 & $(0,7)$ & 3 & $(2,2)$ \\
\hline Não se aplica & 63 & $(46)$ & 4 & $(2,9)$ & 67 & $(48,9)$ \\
\hline \multicolumn{7}{|c|}{ Sofreu abuso sexual } \\
\hline Sim & 10 & $(7,3)$ & 5 & $(3,6)$ & 15 & $(10,9)$ \\
\hline Não & 12 & $(81,8)$ & 10 & $(7,3)$ & 122 & $(89,1)$ \\
\hline \multicolumn{7}{|c|}{ Vítima de crime violento } \\
\hline Sim & 45 & $(32,9)$ & 6 & $(4,4)$ & 51 & $(37,3)$ \\
\hline Não & 77 & $(56,2)$ & 9 & $(6,5)$ & 86 & $(62,7)$ \\
\hline \multicolumn{7}{|c|}{ Esteve em risco de vida } \\
\hline Sim & 66 & $(48,2)$ & 10 & $(7,3)$ & 76 & $(55,5)$ \\
\hline Não & 56 & $(40,9)$ & 5 & $(3,6)$ & 61 & $(44,5)$ \\
\hline
\end{tabular}


A principal substância considerada problema (Tabela 3) foi a Cocaína/Crack em 51,1\% (70) dos participantes, seguida pelo álcool 46,8\% (64). Do total que já consumiram álcool, $77,4 \%$ (106) sentiram pela primeira vez o efeito desta substância com menos de 18 anos. Do total de participantes, 53,3\% (73) apresentavam dificuldades para diminuir ou cessar o uso de SPA, 50,4\% (69) apresentaram sintomas de abstinência e aqueles que conseguiram parar ou diminuir o uso, 69,3\% (95) apresentaram fissuras.

Tabela 3 - Caracterização clínica do consumo de substâncias por pessoas em tratamento. Curitiba, PR, 2018

\section{Variáveis}

\begin{tabular}{cccccc}
\multicolumn{2}{c}{ Masculino } & \multicolumn{2}{c}{ Feminino } & \multicolumn{2}{c}{ Total } \\
\hline $\mathbf{N}$ & $(\%)$ & $\mathbf{N}$ & $(\%)$ & $\mathbf{N}$ & $(\%)$ \\
122 & $(89,1)$ & 15 & $(10,9)$ & 137 & $(100)$
\end{tabular}

SPA considerada problema primário

\begin{tabular}{lcccccc}
\hline Cocaína/Crack & 60 & $(43,8)$ & 10 & $(7,3)$ & 70 & $(51,1)$ \\
\hline Álcool & 59 & $(43,1)$ & 5 & $(3,7)$ & 64 & $(46,8)$ \\
\hline Inalantes & 1 & $(0,7)$ & 0 & $(0)$ & 1 & $(0,7)$ \\
\hline Maconha & 1 & $(0,7)$ & 0 & $(0)$ & 1 & $(0,7)$ \\
\hline Sedativos & 1 & $(0,7)$ & 0 & $(0)$ & 1 & $(0,7)$ \\
\hline Sintomas de Abstinência & & & & & & \\
\hline Não & 38 & $(27,7)$ & 3 & $(2,2)$ & 41 & $(29,9)$ \\
\hline Sim & 62 & $(45,3)$ & 7 & $(5,1)$ & 69 & $(50,4)$ \\
\hline Não se aplica* & 22 & $(16,1)$ & 5 & $(3,6)$ & 27 & $(19,7)$ \\
\hline Dificuldade de dimin
\end{tabular}

Dificuldade de diminuir ou parar de usar SPA

\begin{tabular}{lllllll}
\hline Não & 35 & $(25,5)$ & 2 & $(1,5)$ & 37 & $(27)$ \\
\hline Sim & 65 & $(47,4)$ & 8 & $(5,8)$ & 73 & $(53,3)$ \\
\hline Não se aplica* & 22 & $(16,1)$ & 5 & $(3,6)$ & 27 & $(19,7)$ \\
\hline
\end{tabular}

Fissuras

\begin{tabular}{lllllll}
\hline Não & 37 & $(27)$ & 5 & $(3,6)$ & 42 & $(30,7)$ \\
\hline Sim & 85 & $(62)$ & 10 & $(7,3)$ & 95 & $(69,3)$ \\
\hline Já esteve abstinente por $\geq 1$ ano & & & & & & \\
\hline Não & 45 & $(32,8)$ & 8 & $(5,8)$ & 53 & $(38,6)$ \\
\hline Sim & 42 & $(30,8)$ & 2 & $(1,4)$ & 44 & $(32,2)$ \\
\hline Não responderam & 35 & $(25,5)$ & 5 & $(3,7)$ & 40 & $(29,2)$ \\
\hline
\end{tabular}

Idade que sentiu pela primeira vez o efeito do álcool

\begin{tabular}{lcccccc}
\hline$>18$ & 96 & $(70,1)$ & 10 & $(7,3)$ & 106 & $(77,4)$ \\
\hline $19-25$ & 16 & $(11,7)$ & 1 & $(0,7)$ & 17 & $(12,4)$ \\
\hline$<26$ & 7 & $(5,1)$ & 2 & $(1,5)$ & 9 & $(6,6)$ \\
\hline Não se aplica & 3 & $(2,2)$ & 2 & $(1,5)$ & 5 & $(3,6)$
\end{tabular}

*Resposta válida para aqueles que estavam em uso de substâncias nos últimos seis meses. 
Do total da amostra (Tabela 4), 66,5\% (91) relataram possuir vínculo familiar e 40,9\% (56) mantinham relacionamento amoroso, desses $33,4 \%$ (17) dos parceiros utilizavam SPA e $47 \%$ (24) tinham problemas no relacionamento. Daqueles que possuíam convívio com parentes, 36,4\% (39) apresentaram algum problema no relacionamento. Do total de participantes, $16,1 \%$ (9) tiveram problemas de relacionamento com amigos. Relatos de problemas legais foram 43,8\% (60) histórico de prisão depois dos 18 anos e 20,4\% (28) cometeram furto/roubo.

Tabela 4 - Caracterização familiar, social e legal das pessoas em tratamento. Curitiba, PR, 2018 (continua)

Variáveis

\begin{tabular}{cccccc}
\multicolumn{2}{c}{ Masculino } & \multicolumn{2}{c}{ Feminino } & \multicolumn{2}{c}{ Total } \\
\hline $\mathbf{N}$ & $(\%)$ & $\mathbf{N}$ & $(\%)$ & $\mathbf{N}$ & $(\%)$ \\
122 & $(89,1)$ & 15 & $(10,9)$ & 137 & $(100)$ \\
\hline
\end{tabular}

Vínculo familiar

\begin{tabular}{ccccccc}
\hline $\operatorname{Sim}$ & 81 & $(59,2)$ & 10 & $(7,3)$ & 91 & $(66,5)$ \\
\hline Não & 41 & $29,9)$ & 5 & $(3,6)$ & 46 & $(33,5)$ \\
\hline Total & 122 & $89,1)$ & 15 & $(10,9)$ & 137 & $(100)$
\end{tabular}

Relacionamento amoroso/ parceiro

\begin{tabular}{ccccccc}
\hline Sim & 47 & $34,3)$ & 9 & $(6,6)$ & 56 & $(40,9)$ \\
\hline Não & 75 & $54,8)$ & 6 & $(4,3)$ & 81 & $(59,1)$ \\
\hline Total & 122 & $89,1)$ & 15 & $(10,9)$ & 137 & $(100)$ \\
\hline
\end{tabular}

Parceiro tem problema com SPA ou álcoola

\begin{tabular}{lllllll}
\hline Sim & 11 & $21,6)$ & 6 & $(11,8)$ & 17 & $(33,4)$ \\
\hline Não & 31 & $60,8)$ & 3 & $(5,8)$ & 34 & $(66,6)$ \\
\hline Total & 42 & $82,4)$ & 9 & $(17,6)$ & 51 & $(100)$ \\
\hline
\end{tabular}

Problemas de relacionamento com parceiro ${ }^{\mathrm{a}}$

\begin{tabular}{ccccccc}
\hline Sim & 18 & $35,3)$ & 6 & $(11,7)$ & 24 & $(47)$ \\
\hline Não & 24 & $47,1)$ & 3 & $(5,9)$ & 27 & $(53)$ \\
\hline Total & 42 & $(82,4)$ & 9 & $(17,6)$ & 51 & $(100)$ \\
\hline
\end{tabular}

Problemas de relacionamento com parentes ${ }^{b}$

\begin{tabular}{|c|c|c|c|c|c|c|}
\hline Sim & 36 & $33,6)$ & 3 & $(2,8)$ & 39 & $(36,4)$ \\
\hline Não & 59 & $55,2)$ & 9 & $(8,4)$ & 68 & $(63,6)$ \\
\hline Total & 95 & $88,8)$ & 12 & $(11,2)$ & 107 & $(100)$ \\
\hline \multicolumn{7}{|c|}{ Problemas de relacionamento com amigos ${ }^{c}$} \\
\hline Sim & 9 & $16,1)$ & 0 & (0) & 9 & $(16,1)$ \\
\hline Não & 41 & $73,2)$ & 6 & $(10,7)$ & 47 & $(83,9)$ \\
\hline Total & 50 & $89,3)$ & 6 & $(10,7)$ & 56 & $(100)$ \\
\hline \multicolumn{7}{|c|}{ Preso após 18 anos } \\
\hline Sim & 56 & $40,9)$ & 4 & $(2,9)$ & 60 & $(43,8)$ \\
\hline Não & 66 & $48,2)$ & 11 & (8) & 77 & $(56,2)$ \\
\hline Total & 122 & $89,1)$ & 15 & $(10,9)$ & 137 & $(100)$ \\
\hline
\end{tabular}


Roubou/furtou alguém

\begin{tabular}{ccccccc}
\hline Sim & 28 & $20,4)$ & 0 & $(0)$ & 28 & $(20,4)$ \\
\hline Não & 94 & $68,6)$ & 15 & $(10,9)$ & 109 & $(79,6)$ \\
\hline Total & 122 & $89,1)$ & 15 & $(10,9)$ & 137 & $(100)$
\end{tabular}

Legenda: ${ }^{a} n=86$ não responderam/não aplicável, ${ }^{b} n=30$ não responderam/não aplicável, ${ }^{c} n=81$ não responderam/não aplicável.

A avaliação das subescalas das sete áreas do ASI-6 (Tabela 5) mostra três áreas com escores superiores às demais, representando maior gravidade na área Família/crianças com pontuação de $57,8( \pm 8,6)$, Álcool com 52,8 $( \pm 10,9)$ e Família/problemas Sociais com $51,0( \pm 8,9)$.

Tabela 5 - Escore Addiction Severity Index 6a versão (ASI-6). Curitiba, PR, 2018

\begin{tabular}{lcccccc} 
& \multicolumn{2}{c}{ Veminino } & \multicolumn{2}{c}{ Masculino } & \multicolumn{2}{c}{ Total } \\
\cline { 2 - 7 } & Média & DP & Média & DP & Média & DP \\
\hline Drogas & 44,6 & 11,1 & 43,2 & 10,7 & 44,4 & 11 \\
\hline Família/crianças & 58.3 & 8,6 & 56,1 & 9,1 & 57,8 & 8,6 \\
\hline Álcool & 53.1 & 10,7 & 50,9 & 10,8 & 52,8 & 10,9 \\
\hline Psiquiátrica & 49,7 & 9,4 & 49,2 & 7 & 49,7 & 9,1 \\
\hline Médica & 45 & 9,8 & 47 & 12,5 & 45,2 & 10,1 \\
\hline Legal & 48,2 & 5,1 & 48,1 & 6 & 48,2 & 5,2 \\
\hline Emprego/sustento & 38 & 3,8 & 38,7 & 1,9 & 38,1 & 3,6 \\
\hline Família/apoio social & 37,7 & 6,3 & 37,8 & 8,8 & 37,7 & 6,6 \\
\hline Família/problemas sociais & 50,6 & 8,8 & 50 & 10 & 51 & 8,9
\end{tabular}

Obs: Os SS-Rs foram calculados com base nas instruções fornecidas por Kessler et al. (2012), e então convertidos para escores T.

\section{DISCUSSÃO}

Neste estudo, o predomínio foi do sexo masculino, o que está em concordância com outros estudos brasileiros com pessoas em tratamento no CAPS AD que identificaram o predomínio para o mesmo sexo(7-11). Porém o quantitativo de mulheres pode ser maior ${ }^{(1)}$, alguns fatores podem contribuir para o quantitativo maior de homens pela busca por tratamento, como questões culturais e barreiras sociais ${ }^{(12)}$.

A média de idade neste estudo foi de $41,7( \pm 12,5)$ anos, com maior frequência na faixa etária de 30 a 49 anos, valores aproximados foram encontrados em outros estudos nacionais ${ }^{(8-11,13)}$. Embora o início do uso de SPA geralmente englobe a faixa etária mais 
jovem, a procura para tratamento acontece na fase adulta a partir dos 30 anos, devido aos danos causados e às consequências na vida da pessoa ${ }^{(8)}$.

A maioria dos participantes era solteiro, consoante a outros estudos que ressaltaram que o uso de SPA pode estar associado a vínculos sociais, como companheiros e amigos. Assim, principalmente as mulheres como parceiras destas pessoas são afetadas por constantes discussões, comportamentos agressivos e isolamento social, gerando separações e divórcio (14-15). Tal situação familiar associada ao uso excessivo de SPA agrava mais a condição social do indivíduo, ocasionando o aumento da gravidade dos transtornos relacionados a substâncias ${ }^{(2)}$.

No que concerne à escolaridade, a maioria dos participantes cursou somente o Ensino Fundamental, confirmando que os problemas no processo de aprendizagem desde a infância devido ao uso de SPA podem ser evidenciados na fase adulta, com os elevados números de desemprego ou subemprego. Esse fato pode estar associado aos graves problemas econômicos os quais não afetam somente a pessoa e família, mas também oneram os órgãos públicos como a previdência social, devido ao aumento de afastamento laboral e necessidade de auxílio-doença relacionado aos problemas com o uso de substâncias e suas consequências ${ }^{(8,16)}$.

Referente às condições clínicas agudas e crônicas decorrentes do uso de SPA, em 2018 a Organização Mundial de Saúde divulgou diferentes estratégias para diminuir as mortes e incapacidades geradas pelo álcool, como exemplo, a reformulação na política de comercialização, aumento nos impostos e diminuição de publicidade em mídias sociais. Isso porque, somente no período de 2012 a 2016, o número de mortes no mundo foram de três milhões por esta SPA ${ }^{(17)}$.

Neste estudo, as principais condições clínicas físicas apresentadas foram do sistema circulatório, como a Hipertensão Arterial Sistêmica. Estimativas nacionais realizadas pelo Instituto Nacional de Ciência e Tecnologia para Políticas Públicas do Álcool e Outras Drogas (INPAD), através do LENAD, apontam que as principais consequências físicas entre brasileiros que consomem substâncias são problemas cardiovasculares com 36 milhões (27\%), seguida de neurológicos com 28 milhões $(21,6 \%)$ e imunológicos como processo alérgicos com 27 milhões $(20,5 \%)^{(4)}$. Portanto, entende-se que o aprimoramento de políticas públicas é fundamental para minimizar a gravidade do uso de SPA, pois este agravo é considerado um fator de causa para diversas comorbidades físicas ${ }^{(18)}$.

Com relação à caracterização das condições mentais, os participantes apresentaram manifestações clínicas que levam ao sofrimento psíquico, entre elas destacaram-se a insônia, alterações do humor, agressividade, ideação e tentativa de suicídio. Dados internacionais mostram que 35 milhões de pessoas apresentam transtornos relacionados a substâncias no mundo e necessitam de tratamento(1).

Sabe-se que os eventos traumáticos em sua maioria estão relacionados ao consumo em ambientes vulneráveis como a rua, em decorrência do envolvimento com tráfico de drogas, prática de furto ou roubo para saciar os sintomas de abstinência e compulsão. A falta da substância e a necessidade de consegui-la pode gerar comportamentos de risco, principalmente o crack, por levar a pessoa a uma maior exposição em ambientes vulneráveis, propiciando a vivência de diversos tipos de violência, gerando consequências como traumas ${ }^{(2)}$.

Quanto aos sintomas de fissura e abstinência, os dados da presente pesquisa estão em consonância com um estudo realizado com 160 pessoas que faziam uso de cocaína e crack, dessas, 109 apresentaram sintomas de abstinência nos últimos 30 dias e 124 sintomas de fissura. Os que apresentaram sintomas mais intensos de fissura e abstinência foram os usuários de crack com 51,6\% (56) e 52,4\% (65) respectivamente ${ }^{(18)}$. O crack é usado pela via inalatória e sua ação ocorre diretamente no sistema respiratório, sendo seu efeito menos duradouro que a cocaína inalada. Portanto, devido ao efeito rápido com curta duração e tolerância, ocorre o aumento da frequência de uso, aumentando a gravidade do transtorno(3). 
Verificou-se neste estudo que o primeiro efeito sentido pelo uso do álcool foi com menos de 18 anos. A literatura aborda que o início do uso dessa SPA acontece principalmente na adolescência, considerada a fase de desenvolvimento para a adulta, porém, com o início precoce de uso de SPA essa fase pode ser prejudicada, gerando consequências como transtornos mentais, problemas profissionais e familiares ao longo da vida(10).

Neste estudo, a área de maior gravidade do uso de SPA com o escore mais elevado foi Família/criança, devendo ser valorizada a de terceiro escore que se refere a Família/ problemas sociais, confirmando que os problemas de relacionamentos com a família e sociedade para a pessoa que usa SPA são marcadamente graves. Um estudo que avaliou o nível de gravidade utilizando o ASI em seis capitais do Brasil, com 740 participantes, apresentou maior escore na região Sul na área Família/criança 52.0 ( \pm 7.7$)$ e Família/ problemas sociais $56.7( \pm 9.5)^{(19)}$.

Ressalta-se que a criança sofre os efeitos do uso de SPA pelos seus genitores desde o desenvolvimento intrauterino, influenciando seu comportamento e aspectos psicológicos. Os principais problemas identificados são vulnerabilidade da criança em ambientes de violência física e sexual, comorbidades físicas e psiquiátricas e perda de guarda dos pais ${ }^{(20)}$. Um estudo mostrou que crianças que estavam em orfanatos tinham menos problemas comportamentais do que crianças que viviam com familiares com transtornos relacionados a substâncias ${ }^{(21)}$.

A segunda área de maior gravidade neste estudo foi a álcool com média $52.8( \pm 10,9)$. No estudo citado anteriormente, a área de maior gravidade nas seis capitais do Brasil foi o Álcool $55.8( \pm 10,3)$, sendo a maior média na cidade de São Paulo com $57.3( \pm 9.8)^{(19)}$. Esses resultados podem estar relacionados aos aspectos culturais do consumo de álcool na história da sociedade, uma SPA lícita e seu uso considerado natural no meio social. O álcool é considerado a causa de mais 200 doenças e lesões, e o uso dessa SPA está associado a outros transtornos mentais e infecções sexualmente transmissíveis, além dos prejuízos sociais e econômicos ${ }^{(22)}$.

Existem problemas, como comportamentos de risco, que podem estar relacionados com a intoxicação aguda do álcool, que altera o comportamento e dificulta o discernimento dos riscos iminentes a sua vida ${ }^{(3,22)}$. Outro aspecto prejudicial é o fato do álcool ser considerado SPA lícita, o que pode favorecer o início precoce do uso, a dificuldade de manter a abstinência e também a descontinuidade do tratamento, sendo assim, frequentemente, desconsideradas as consequências causadas pelo álcool(21,22).

Este estudo apresenta limitações quanto à amostragem por conveniência, considerando que aqueles usuários inseridos no CAPS e que não compareceram no serviço no período da coleta de dados não participaram da pesquisa. Ademais, a utilização de um instrumento de autorretrato possibilita subestimar ou superestimar os dados coletados.

\section{CONSIDERAÇÕES FINAIS}

O objetivo deste estudo foi atingido ao possibilitar a descrição multidimensional e as áreas de maior gravidade dos transtornos relacionados a substâncias na vida de pessoas em tratamento nos Centros de Atenção Psicossocial Álcool e outras Drogas. Essa gravidade foi observada nos aspectos familiares, sociais, demográficos e econômicos, físicos e psicológicos e psiquiátricos.

As áreas que apresentaram maiores escores de gravidade dos transtornos relacionados a substâncias foram Família/Criança, Família/Problemas sociais e Álcool, reforçando dois importantes aspectos: o primeiro de que as relações familiares e sociais são afetadas pelo uso de SPA, e o segundo que o álcool se configura como uma droga lícita, de fácil acesso e presente de modo geral na vida das pessoas desde a infância, por vezes provando-a com 
a permissão dos adultos, e também por observar seu uso, acaba sendo a SPA de maior frequência entre todas as faixas etárias.

Entende-se que tais resultados poderão estimular os profissionais de saúde a conhecer os principais problemas sociais e de saúde física e mental das pessoas com transtornos relacionados a substâncias, bem como a organização do cuidado dos profissionais da saúde a esta clientela e sua família.

\section{AGRADECIMENTOS}

Ao Conselho Nacional de Desenvolvimento Científico e Tecnológico (CNPq) pelo incentivo financeiro de bolsa produtividade à orientadora deste trabalho, sob processo: 308045/2015-7, chamada: PQ 2015, Projeto: Adesão ao tratamento pelo dependente de substância psicoativa.

\section{REFERÊNCIAS}

1. United Nations Office on Drugs and Crime (UNODC). World Drug Report 2019 [Internet]. Vienna: United Nations Office on Drugs and Crime; 2019.

2. Halpern SC, Scherer JN, Faller S, Sordi A, Ornell F, Dalbosco C, et al. Vulnerabilidades clínicas e sociais em usuários de crack de acordo com a situação de moradia: um estudo multicêntrico de seis capitais brasileiras. Cad. Saúde Pública [Internet]. 2017 [acesso em 13 nov 2019]; 33(6). Disponível em: http:// dx.doi.org/10.1590/0102-311x00037517.

3. Sadock BJ, Sadock VA, Ruiz P. Compêndio de psiquiatria: ciência do comportamento e psiquiatria clínica. 11. ed. Porto Alegre: Artmed; 2017.

4. Instituto Nacional de Ciência e Tecnologia para Políticas Públicas do Álcool e Outras Drogas (INPAD). II Levantamento Nacional de Álcool e Drogas (LENAD). São Paulo: Instituto Nacional de Ciência e Tecnologia para Políticas Públicas de Álcool e Outras Drogas; UNIFESP; 2014.

5. Kessler F, Cacciola J, Alterman A, Faller S, Souza-Formigoni ML, Cruz MS, et al. Psychometric properties of the sixth version of the Addiction Severity Index (ASI-6) in Brazil. Rev Bras Psiquiatr. [Internet]. 2012 [acesso em 00 mês abreviado 0000]; 34(1). Disponível em: http://dx.doi.org/10.1590/ S1516-44462012000100006.

6. McLellan AT, Luborsky L, Woody GE, O'Brien CP. An improved diagnostic evaluation instrument for substance abuse patients: The Addiction Severity Index. J Nerv Ment Dis. [Internet]. 1980 [acesso em 00 mês abreviado 0000]; 168(1). Disponível em: https://www.ncbi.nlm.nih.gov/pubmed/7351540.

7. Câmara HS, Martins MLB. Uso de substâncias psicoativas e perfil nutricional de usuários do CAPS AD III, Palmas/TO. Rev Ciênc Ext. [Internet]. 2017 [acesso em 09 maio 2019]; 13(1). Disponível em: https://ojs. unesp.br/index.php/revista_proex/article/view/1397/134.

8. Oliveira VC de, Capistrano FC, Ferreira ACZ, Kalinke LP, Felix JVC, Maftum MA. Perfil sociodemográfico e clínico de pessoas atendidas em um CAPS AD do sul do Brasil. Rev Baiana Enferm. [Internet]. 2017 [acesso em 18 fev 2020]; 31(1). Disponível em: http://dx.doi.org/10.18471/rbe. v31i1.16350.

9. Capistrano FC, Maftum GJ, Mantovani M de F, Felix, JVC, Kalinke, LP, Nimtz MA, Maftum MA. Consequência do uso abusivo de substâncias psicoativas por pessoas em tratamento. Saúde e pesq. [Internet]. 2018 [acesso em 25 maio 2018]; 11(1). Disponível em: https://pesquisa.bvsalud.org/enfermeria/ resource/pt/biblio-884289. 
10. Conceição DS, Andreoli SB, Esperidião MA, Santos DN dos. Attendance of children and adolescents with psychoactive substance use disorders performed by Psychosocial Care Centers in Brazil, 2008-2012. Epidemiol. Serv. Saúde [Internet]. 2018 [acesso em 13 nov 2019]; 27(2). Disponível em: http://dx.doi. org/10.5123/s1679-49742018000200002.

11. Trevisan ER, Castro S de S. Centros de Atenção Psicossocial - álcool e drogas: perfil dos usuários. Saúde debate [Internet]. 2019 [acesso em 13 nov 2019]; 43(121). Disponível em: http://dx.doi. org/10.1590/0103-1104201912113.

12. Albuquerque $C$ de $S$, Nóbrega $M$ do PSS. Barreiras e facilidades encontradas por mulheres usuárias de substâncias psicoativas na busca por tratamento especializado. SMAD. Rev Eletrônica Saúde Mental Álcool Drog. [Internet]. 2016 [acesso em 03 dez 2019]; 12(1). Disponível em: https://dx.doi.org/10.11606/ issn.1806-6976.v12i1p22-29.

13. Borba L de O, Maftum MA, Vayego SA, Kalinke LP, Ferreira ACZ, Capistrano FC. Perfil do portador de transtorno mental em tratamento no CAPS. REME - Rev Min Enferm. [Internet]. 2017 [acesso em 03 dez 2019]; 21(e1010). Disponível em: http://www.dx.doi.org/10.5935/1415-2762.20170020.

14. Oliveira MVP de, Nunes EPO, Nunes JR. Reinserção profissional da mulher em tratamento no CAPS AD III em Palmas -TO. Rev Humanid Inov. [Internet]. 2019 [acesso em 18 fev 2020]; 6(12). Disponível em: https://revista.unitins.br/index.php/humanidadeseinovacao/article/view/1242.

15. Mota WH, Silva FC, Soares WKR, Kuster DE, Medeiros PC, Siqueira RP, et al. Comorbidades associadas ao uso de drogas em usuários que se submeteram ao tratamento em comunidade terapêutica de Cacoal-RO. In: Anais do Congresso da Sociedade Brasileira de Medicina Tropical. [Internet]. Campinas: Galoá; 2018 [acesso em 18 fev 2020]. Disponível em: https://proceedings.science/medtrop/papers/ comorbidades-associadas-ao-uso-de-drogas-em-usuarios-que-se-submeteram-ao-tratamento-emcomunidade-terapeutica-de-cacoal\#.

16. Fernandes MA, Santos JDM, Moraes LMV de, Lima JSR, Feitosa CDA, Sousa LFC. Mental and behavioral disorders in workers: a study on work leave. Rev. esc. enferm. USP. [Internet]. 2018 [acesso em 18 fev 2020]; 52(e03396). Disponível em: http://dx.doi.org/10.1590/S1980-220X2017036403396.

17. World Health Organization (WHO). Global status report on alcohol and health 2018. Geneva: World Health Organization; 2018

18. Santos, JAT. Gravidade da dependência de cocaína (Fumada e Inalada) em indivíduos em tratamento ambulatorial. [tese]. Ribeirão Preto (SP): Universidade de São Paulo; 2017.

19. Faller S, Peuker AC, Sordi A, Stolf A, Souza-Formigoni ML, Cruz MS, et al. Who seeks public treatment for substance abuse in Brazil? Results of a multicenter study involving four Brazilian state capitals. Trends Psychiatry Psychother [Internet]. 2014 [acesso em 18 fev 2020]; 36(4). Disponível em: https://doi.org/10.1590/2237-6089-2014-0040.

20. Campelo LL de CR, Santos RC de A, Angelo M, Nóbrega M do PS de S. Efeitos do consumo de drogas parental no desenvolvimento e saúde mental da criança: revisão integrativa. SMAD, Rev. Eletrônica Saúde Mental Álcool Drog. [Internet]. 2018 [acesso em 18 fev 2020]; 14(4). Disponível em: https://dx.doi.org/10.11606/issn.1806-6976.smad.2018.000411.

21. Hjerkinn B, Lindbæk M, Rosvold EO. Behaviour among children of substance-abusing women attending a Special Child Welfare Clinic in Norway, as assessed by Child Behavior Checklist (CBCL). Scand J Caring Sci. [Internet]. 2012 [acesso em 18 fev 2020]; 27(2). Disponível em: https://doi.org/10.1111/ j.1471-6712.2012.01030.x.

22. Antunes A, Costa ECV. Sono, agressividade e comportamentos de risco em indivíduos dependentes de drogas e de álcool. Anál. psicol. [Internet]. 2019 [acesso em 18 fev 2020]; 37(1). Disponível: https:// dx.doi.org/10.14417/ap.1390. 
Recebido: 10/12/2019

Finalizado: 15/07/2020

\author{
Autor Correspondente: \\ Manuela Kaled \\ Universidade Federal do Paraná \\ R. Lothário Meisser, 632 - 80210-170 - Curitiba, PR, Brasil \\ E-mail: manuelakaled@gmail.com
}

\title{
Contribuição dos autores:
}

Contribuições substanciais para a concepção ou desenho do estudo; ou a aquisição, análise ou interpretação de dados do estudo - FCC, ACZF, CBA

Elaboração e revisão crítica do conteúdo intelectual do estudo - FCC, ACZF, MAM

Aprovação da versão final do estudo a ser publicado - FCC, ACZF, MAM, MFM, RDCMP, CBA

Responsável por todos os aspectos do estudo, assegurando as questões de precisão ou integridade de qualquer parte do estudo - MK

\section{(c) (4)}

Este obra está licenciado com uma Licença Creative Commons Atribuição 4.0 Internacional. 